\section{Programme FMH "Gestion de la qualité»}

Sous l'égide de la FMH, l'Institut universitaire de la médecine sociale et préventive (IUMSP), Lausanne, propose une série de séminaires à l'intention du corps médical sur la gestion de la qualité.

\section{Contenu}

Séminaire de base

Cadre juridique de la gestion de la qualité; concepts et instruments de la qualité en général; concepts et mesures de la qualité des soins médicaux; «Evidencebased Practice" (évaluation et critique de données et d'études); base des évaluations coût/efficacité; internet et gestion de la qualité.

Séminaire avancé I

Bases de l'économie de la santé; principes de l'épidémiologie clinique et de l'«Evidence-based Medicine»; Guidelines (recommandations pour la pratique clinique) et l'évaluation de leur qualité; cercles de qualité; planification et mise en œuvre de projets de gestion de la qualité; exemples de projets de gestion de la qualité réussis; mesures de la satisfaction des patients; échange d'expériences; banques de données se rapportant à la qualité des soins.

\section{Séminaire avancé II}

L'adéquation des interventions diagnostiquées et thérapeutiques (théorie et pratique); qualité de la prescription médicamenteuse; promotion de la qualité dans le domaine de la prévention; évaluation de la qualité des résultats en termes de santé; accréditation et certification dans le domaine de la santé; convention relatives à la qualité dans le cadre de la LAMal; prévention, évaluation et gestion des erreurs médicales; examen final.

\section{Participants}

Le programme s'adresse aux médecins, quelle que soit leur domaine d'activité. Chaque séminaire comprendra un minimum de 12 et un maximum de 24 participants.

\section{Méthode didactique}

Tant le séminaire de base que les séminaires avancés seront conduits sous une forme d'apprentissage interactif en lien avec les questions et les expériences des participants. Des nouvelles connaissances et capacités seront développées par l'analyse de projets achevés et en élaborant des projets pertinents pour le domaine d'activité des participants.

\section{Certificat}

La participation au séminaire de base et aux séminaires avancés sera sanctionnée par une attestation de participation qui pourrait compter, en partie, pour une future attestation de formation complémentaire (AFC).

\section{Responsables}

PD Dr John-Paul Vader et PD Dr Bernard Burnand (IUMSP, Lausanne)

Dr Hans-Heinrich Brunner, Président FMH

\section{Dates, lieu et coûts}

Séminaire de base

$$
31 \text { mars } 2001 \quad 1 \text { journée }
$$

Séminaire avancé I

$$
29 \text { et } 30 \text { juin } 2001 \quad 2 \text { jours }
$$

Séminaire avancé II

$$
27 \text { au } 29 \text { septembre } 20013 \text { jours }
$$

Lieu: Lausanne ou environs

Coûts (comprenant le séminaire, documentation, repas de midi, pauses cafés):

- Séminaire de base

Fr. 700.-

- Séminaires avancés Fr. 2000.-

\section{Inscription}

Institut universitaire de médecine sociale et préventive, Rue du Bugnon 17, 1005 Lausanne, Mme Claude Mühlemann, tél. 02131472 62, fax 02131449 54, e-mail claude.muhlemann@hospvd.ch

Délai d'inscription: 28 février 2001 